\title{
Analysis of Dynamic System Reliability Based on Adaptive Response Surface Method
}

\author{
Wei SHEN*, Qingbin ZHANG, Wenliang FAN \\ School of Civil Engineering \\ Chongqing University \\ Chongqing, China \\ e-mail: cosprit@163.com
}

\begin{abstract}
Dynamic system reliability is one of the topics for structure reliability analysis. However, the classical system reliability and dynamic system reliability often have multiple failure modes which lead to the combinatorial explosion problems. In this paper, a new method for analyzing the reliability of dynamic system reliability is proposed. Firstly, based on the level of load and dynamic condition, the limit state function for each failure modes are available. Secondly, by introducing the equivalent extreme value event, the equivalent performance function for individual limit state function is derived. Thirdly, the adaptive RSM is proposed to analyze the reliability index of the structure system under dynamic condition. Finally, several examples are presented to illustrate the accuracy and efficiency of the proposed method.
\end{abstract}

Keywords-dynamic system reliability; failure mode; limit state function; equivalent extreme value event; adaptive response surface method

\section{INTRODUCTION}

In structural reliability analysis, the work and studies with respect to dynamic system reliability analysis are concerning and developed for decades because of the complexity and inevitability of randomness both in structural system and dynamic load, and a number of probabilistic analysis tools have been proposed to quantify dynamic system reliability analysis: In system reliability analysis, Ang [1] first introduced the idea of fault tree analysis (FTA) into structural system reliability analysis and proposed the probabilistic network evaluation technique (PENT) algorithm for evaluating the comprehensive failure probability of structural system. Then Mose [10] combined the incremental load approach and limit state analysis method for identifying and expressing the main collapse modes in structural system reliability analysis. ThoftChristensen \& Sorensen [13] presented $\beta$-unzipping method for calculating the probability failure of series and parallel structural systems, which the lower-upper bound method is applied. Murotsu \& Thoft-Christensen [14] modified the selection of most significant failure modes by introducing joint failure probability, and proposed branch- and bound method for estimating the reliability of structural system. Feng \& Mose [7] introduced definitions for redundancy based on ultimate, reserve, and residual strengths in the context of structural reliability and optimum design of truss structures. As for dynamic aspects, Rice [12] first proposed the formulation of the out-crossing theory and it is widely developed and applied [4]. However, the major difficulties encountered in all these dynamic system reliability methods are how to solve the combinatorial explosion problems in multiple failure modes and how to tackle the use of correlation information of different random events and twodimensional joint probability density function (PDF). In order to overcome these difficulties, J. Li [9] proposed the equivalent extreme value event method for dynamic reliability analysis to derive the exact solution with inherent total correlation information.

Response surface method (RSM) is a helpful technique in structural reliability analysis where limit state function is implicit and numerical methods are needed. In RSM, the limit state function is approximated by mathematical expression with undetermined coefficients. By fitting the response surface to a number of sample points on the limit state, the response surface function (RSF) is constructed and applied in reliability analysis, such as first-order reliability method (FORM) [8] and second-order reliability method (SORM) [2]. The selection of the form of RSF has great influence on the efficiency and accuracy of RSM, and the polynomial form of RSF is commonly used and studied [3, 6, 11, 15], and Fan [5] proposed an adaptive response surface method with cross terms, which the cross terms can be rationally considered and improve the efficiency of RSM without loss of accuracy.

In this paper, an adaptive RSM is presented for dynamic system reliability analysis. It is organized as follows. In Section 2, an equivalent performance function for dynamic structural reliability is formulated based on the equivalent extreme value event, and an adaptive RSM is presented based on the equivalent performance function together with its implementation. Then in Section 3, an examples are investigated to verify the proposed method. At last some conclusion are drawn in Section 4.

\section{ADAPTIVE RESPONSE SURFACE METHOD FOR DYNAMIC RELIABILITY ANALYSIS}

\section{A. The equivalent extreme-value event}

For the first-passage problem, if there are multiple limit state functions in dynamic reliability analysis, the reliability probability can be defined as 


$$
P_{f}=\operatorname{Pr}\{\overbrace{i=1}^{m}\left(g_{i}(X, t)>0\right), t \in\left[0, T_{i}\right]\}
$$

where $\operatorname{Pr}\{\cdot\}$ is the probability of the random event in the bracket; $g_{i}(\cdot)$ is the $i$ th time dependent limit state function; $\boldsymbol{X}=\left(X_{1}, X_{2}, \ldots, X_{n}\right)$ is the random vector with $n$ components, and $T_{i}$ is the time duration corresponding to $g_{i}(\cdot)$.

Introducing the equivalent extreme-value event [9], (1) can be rewritten as

$$
\begin{aligned}
& P_{f}=\operatorname{Pr}\left\{\min _{1 \leq i \leq m}\left(\min _{t \in\left[0, T_{i}\right]}\left(g_{i}(\boldsymbol{X}, t)\right)\right)>0\right\} \\
& =\operatorname{Pr}\left\{g_{e q}(\boldsymbol{X}, \boldsymbol{T})>0\right\}
\end{aligned}
$$

where $g_{e q}(\cdot)$ is the equivalent limit state function, namely

$$
g_{e q}(\boldsymbol{X}, \boldsymbol{T})=\min _{1 \leq i \leq m}\left(\min _{t \in\left[0, T_{i}\right]}\left(g_{i}(\boldsymbol{X}, t)\right)\right)
$$

where $\boldsymbol{T}=\left(T_{1}, \ldots, T_{m}\right)$.

Therefore, the multiple limit state functions in dynamic system reliability are translated into one equivalent limit state function, which can be approximated by RSF efficiently.

\section{B. Adaptive response surface method}

Based on the quadratic polynomial response surface method (RSM), the equivalent limit state function $g_{e q}(\boldsymbol{X}, \boldsymbol{T})$ can be approximated by

$$
\begin{aligned}
& g_{e q}(\boldsymbol{X}, \boldsymbol{T}) \approx \bar{g}_{e q}(\boldsymbol{X}, \boldsymbol{T})= \\
& a_{0}+\sum_{i=1}^{n} b_{i} X_{i}+\sum_{i=1}^{n} c_{i} X_{i}{ }^{2}+\sum_{1 \leq i<j \leq n} d_{i j} X_{i} X_{j}
\end{aligned}
$$

where $a_{0}, b_{i}, c_{i}$ and $d_{i j}$ are the $1+2 n+n(n-1) / 2$ undetermined coefficients, which can be evaluated by a set of linear equations through the selecting sample points.

However, not all the cross terms for each pair of $\left(X_{i}, X_{j}\right)$ are certainly necessary in practice. Introducing an indication function $I\left(X_{i}, X_{j} \boldsymbol{T}\right)$ for the cooperative effects between $X_{i}$ and $X_{j}$, say

$$
I\left(X_{i}, X_{j}, \boldsymbol{T}\right)=\left\{\begin{array}{l}
1, \text { if the cross term sof } X_{i} \text { and } X_{j} \text { exist } \\
0, \quad \text { otherwise }
\end{array}\right.
$$

which can be determined according to [5], and (4) is rewritten further as

$$
\begin{aligned}
& \bar{g}_{e q}(\boldsymbol{X}, \boldsymbol{T})=a_{0}+\sum_{i=1}^{n} b_{i} X_{i}+\sum_{i=1}^{n} c_{i} X_{i}{ }^{2} \\
& +\sum_{1 \leq i<j \leq n} I\left(X_{i}, X_{j}, \boldsymbol{T}\right) d_{i j} X_{i} X_{j}
\end{aligned}
$$

Due to involving cross terms rationally, (6) is named as the adaptive RSM. Based on (6), the dynamic system reliability index $\beta$ can be obtained easily by Monte Carlo simulation (MCS).

\section{Numerical implementation}

The procedure of the proposed method is as follows:

1) The initial iterative analysis

a) Set $k=0$, and $\boldsymbol{x}_{c}=\left(x_{1, c}, x_{2, c}, \ldots, x_{n, c}\right)=\boldsymbol{\mu}_{x}$, in which $\boldsymbol{x}_{c}$ is the reference point and $\boldsymbol{\mu}_{x}$ is the mean value of $\boldsymbol{X}$.

b) By selecting sample points along the axes $x_{i}$ as the point at $\boldsymbol{x}_{c}$ and points with coordinates $x_{i}^{(0)}=x_{i, c} \pm f_{k} \sigma_{i}$, (if $k=0$, then $f_{k}=3$, else $f_{k}=1$ ), in which $\sigma_{i}$ is the standard deviation of variable $X_{i}$. And evaluate the function values of $g_{e q}(\boldsymbol{X}, \boldsymbol{T})$ at these sample points, $2 n+1$ linear equations can be obtained.

c) By selecting sample points at $\left(x_{i, c}+f_{k} \sigma_{i}, x_{j, c}+f_{k} \sigma_{j}, \underline{\boldsymbol{x}}_{i j, c}\right)$, $(1 \leq i<j \leq n)$, in which $\underline{\boldsymbol{x}}_{i j, c}$ is a sub-vector of $\boldsymbol{x}_{c}$ without the corresponding coordinates of $X_{i}$ and $X_{j}$. And evaluate the function values of $g_{e q}(\boldsymbol{X}, \boldsymbol{T})$ at these sample points, $n(n-1) / 2$ equations can be obtained.

d) According to reference [5], $\Delta g_{i j}{ }^{(0)}$ can be evaluated using the results of sub-step (a) and (b), reads:

$$
\Delta g_{i j}{ }^{(0)}=\frac{\left|\Delta g_{e q}{ }^{(1)}-\Delta g_{e q}{ }^{(2)}\right|}{\min \left\{\left|\Delta g_{e q}{ }^{(1)}\right|,\left|\Delta g_{e q}{ }^{(2)}\right|\right\}}
$$

where

$$
\left\{\begin{array}{l}
\Delta g_{e q}{ }^{(1)}=g_{e q}\left(x_{i, 1}, \underline{x}_{i, c}, \boldsymbol{T}\right)-g_{e q}\left(\boldsymbol{x}_{c}, \boldsymbol{T}\right) \\
\Delta g_{e q}{ }^{(2)}=g_{e q}\left(x_{i, 1}, x_{j, 1}, \underline{\boldsymbol{x}}_{i j, c}, \boldsymbol{T}\right) \\
-g_{e q}\left(x_{j, 1}, \underline{\boldsymbol{x}}_{j, c}, \boldsymbol{T}\right)
\end{array}\right.
$$

and $x_{i, 1}=x_{i, c}+f_{k} \sigma_{i}$ and $x_{j, 1}=x_{j, c}+f_{k} \sigma_{j}$. Then the indication functions $I^{(0)}\left(X_{i}, X_{j}\right)(1 \leq i<j \leq n)$ can be determined by

$$
I^{(0)}\left(X_{i}, X_{j}\right)=\left\{\begin{array}{l}
1, \text { if } \Delta g_{i j}{ }^{(0)}>\varepsilon \\
0, \text { otherwise }
\end{array}\right.
$$


where $\varepsilon$ is a specific positive value and $\varepsilon=0.001$ in this work.

e) If $I^{(0)}\left(X_{i}, X_{j}\right)^{(0)}=1$, the cross term of $X_{i}$ and $X_{j}$ is existed in (6). Then the undetermined coefficients $a_{0}, b_{i}, c_{i}$ and $d_{i j}$ can be obtained by solving the resulting simultaneous equations in sub-step (b) and (c), and yields the initial response surface, noted as $\bar{g}_{e q}{ }^{(0)}(\boldsymbol{X}, \boldsymbol{T})$, and the reliability index $\beta^{(0)}$ of $\bar{g}_{e q}{ }^{(0)}(\boldsymbol{X}, \boldsymbol{T})$ can be obtained by the first-order reliability method(FORM), together with the design point $\boldsymbol{x}^{*(0)}$.

f) Set $k=1$, and select a new center point $\boldsymbol{X}_{M}{ }^{(1)}$ for the 1 st iteration using the linear interpolation

$$
\boldsymbol{x}_{M}^{(k)}=\boldsymbol{\mu}_{x}+\left(\boldsymbol{x}^{*(k-1)}-\boldsymbol{\mu}_{X}\right) \frac{g\left(\boldsymbol{\mu}_{x}, \boldsymbol{T}\right)}{g\left(\boldsymbol{\mu}_{x}, \boldsymbol{T}\right)-g\left(\boldsymbol{x}^{*(k-1)}, \boldsymbol{T}\right)}
$$

g) Let $\boldsymbol{x}_{c}=\boldsymbol{x}_{M}{ }^{(1)}$. Being similar with the process of $k=0$, $I^{(1)}\left(X_{i}, X_{j}, \boldsymbol{T}\right), \beta^{(1)}$ and $\boldsymbol{x}^{*(1)}$ are available easily.

2) Delineating the existence of bivariate component function

According to the results of step (1), if both $I^{(0)}\left(X_{i}\right.$, $\left.X_{j}, \boldsymbol{T}\right)$ and $I^{(1)}\left(X_{i}, X_{j}, \boldsymbol{T}\right)$ are equal to 0 , then $I\left(X_{i}, X_{j}\right)=0$, otherwise there exists cross terms of $X_{i}$ and $X_{j}$ in (6).

3) Subsequent iterative analysis

i) If $\left|\beta^{(k)}-\beta^{(k-1)}\right| /\left|\beta^{(k-1)}\right| \leq \varepsilon_{3}\left(\varepsilon_{3}=10^{-3}\right)$, go to sub-step vi); or else, go to sub-step ii).

ii) Set $k=k+1$, and $\boldsymbol{x}_{c}=\boldsymbol{X}_{M}{ }^{(\mathrm{k})}$, which is determined by Eq. (10).

iii) Selecting $2 n+1$ sample points along the axes of $x_{i}$ which are located at $\boldsymbol{x}_{c}$ and with coordinates $x_{i}^{(k)}=$ $x_{i, c} \pm f_{k} \sigma_{i}$, and evaluate the function values of $g_{e q}(\boldsymbol{X}$, T) at these sample points.

iv) If $I\left(X_{i}, X_{j}\right)=1$ for $k \geq 2$, select an additional point $\left(x_{i, c}+f_{k} \sigma_{i}, x_{j, c}+f_{k} \sigma_{j}, \underline{\boldsymbol{x}}_{i j, c}\right)$ and evaluate its function value of $g_{e q}(\boldsymbol{X}, \boldsymbol{T})$.

v) Evaluate the undetermined coefficients $a_{0}, b_{i}, c_{i}$ and $d_{i j}$ by solving the resulting simultaneous equations in sub-step (iii) and (iv), and yields the $k$ th response surface, namely $\bar{g}_{e q}{ }^{(\mathrm{k})}(\boldsymbol{X}, \boldsymbol{T})$. Then the corresponding reliability index $\beta^{(k)}$ can be obtained by FORM, together with the corresponding design point $\boldsymbol{X}^{*(k)}$. And go to sub-step i).

vi) Estimate the failure probability associated with $\bar{g}$ $e^{(\mathrm{k})}(\boldsymbol{X}, \boldsymbol{T})$ using Monte Carlo simulation (MCS).

\section{NUMERICAL EXAMPLES: IDEAL ELASTIC- PLASTIC ONE-BAY EIGHT-STORY FRAME}

In order to demonstrate the performance of this method, the dynamic system reliability of a one bay eight-story ideal elastic-plastic steel frame structure is investigated, which is constituted by beam elements. The geometrical properties of the structure and beam cross section are shown in Figure 1 and Figure 2. The initial Young's modulus E, the ultimate stress of all sections $f$ and the amplification coefficient of ground acceleration $A$ are taken as random variables, and their statistics are listed in Table 1. And the Tafat stochastic ground motion model is used.

The reliabilities agianst the ith inter-story drift can be described as

$$
R_{i}=\operatorname{Pr}\left\{X_{i}(t) / h_{i}<\phi_{B}, t \in\left[0, T_{i}\right]\right\} \quad(i=1,2, \cdots, 8)
$$

where $X_{i}(i=1,2, \ldots, 8)$ is the inter-story drift between the (i1)th story and the $i$ th story, $h_{i}$ being the corrseponding height, $\phi_{B}$ being the threshold of the inter-story drift angle. In this example, $\phi_{B}$ is defined as $1 / 50$ for safety under the seldom occured earthquake. Besides, the dynamic system reliability can be defined as

$$
R=\operatorname{Pr}\left\{\bigcap_{i=1}^{\&}\left(X_{i}(t) / h_{i}<\phi_{B}\right), t \in\left[0, T_{i}\right]\right\}
$$

and the equivalent limit state function $g_{e q}(f, A, E, \boldsymbol{T})$ can be described as

$$
g_{\text {eq }}(f, A, E, \boldsymbol{T})=\min _{1 \leq i \leq 8}\left[1-\max _{t \in T_{i}}\left(X_{i}(f, A, E, t) / h_{i} \phi_{B}\right)\right]
$$

where $\boldsymbol{T}=\left(T_{1}, T_{2}, \ldots, T_{8}\right)$.

By delineating all the cross terms, the results showed that $I^{(0)}(f, A, \boldsymbol{T})=I^{(1)}(f, A, \boldsymbol{T})=I^{(0)}(f, E, \boldsymbol{T})=I^{(1)}(f, E, \boldsymbol{T})=0$. Then the response surface function can be written as

$$
\begin{aligned}
& \bar{g}_{\mathrm{eq}}(f, A, E, \boldsymbol{T})=a_{0}+b_{1} f+b_{2} A+ \\
& b_{3} E+c_{1} f^{2}+c_{2} A^{2}+c_{3} E^{2}+d_{A E} A E
\end{aligned}
$$

After 8 iterations the convergent result can be obtained, and the final reliability index $\beta$ is 3.0436 . The value of $\beta$ obtained by Monte Carlo simulation of 1000000 sample size is 2.8018 .

TABLE I. STATISTICS OF RANDOM VARIABLES

\begin{tabular}{|c|c|c|c|}
\hline Variable & Distribution & $\begin{array}{c}\text { Mean } \\
\text { value }\end{array}$ & $\begin{array}{c}\text { Standard } \\
\text { deviation }\end{array}$ \\
\hline$f(M p a)$ & Normal & 400 & 40 \\
\hline$A$ & Normal & 1 & 0.3 \\
\hline$E(M p a)$ & normal & $2.0 \mathrm{e} 05$ & $2.0 \mathrm{e} 04$ \\
\hline
\end{tabular}




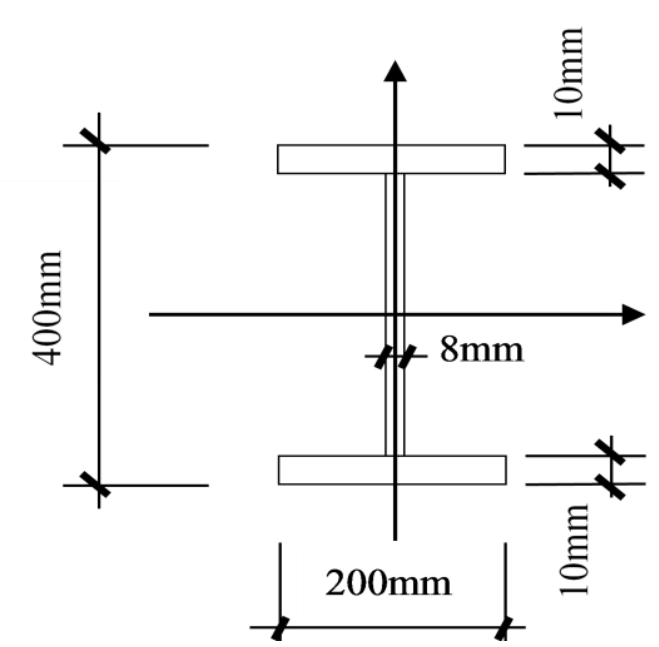

Figure 1. Geometrical properties of beam cross section.

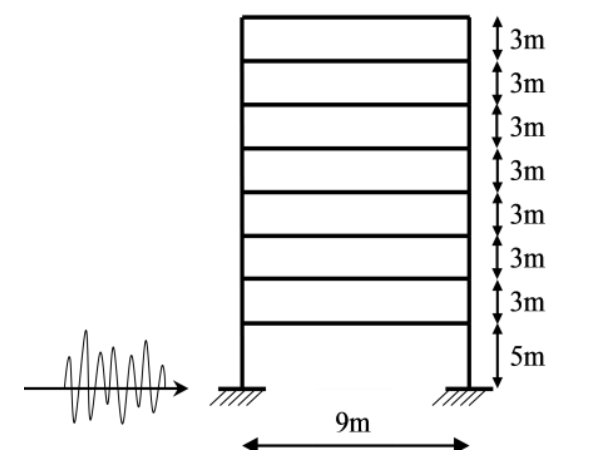

Figure 2. Geometrical properties of the structure.

\section{CONCLUSION}

Structural dynamic reliability analysis has been a significant subject in the area of structural reliability analysis. In this work, an adaptive RSM for dynamic reliability analysis is proposed by combining the equivalent extreme value event with delineating the existence of cross terms, which the cross terms in a quadratic response surface function can be rationally considered, and better balancing the accuracy and efficiency of the iterative RSM. Then the MCS is applied to obtain the final reliability index $\beta$. A multi-story steel frame example is investigated to demonstrate the effectiveness of the proposed method.

\section{ACKNOWLEDGMENT}

The research reported in this paper was conducted with the support of the National Natural Science Foundation of China (Grant No. 51678092) and Construction and Technology Program of Chongqing, China (Grant No. 20160179).

\section{REFERENCES}

[1] A. H-S. Ang, J. Abdelnou and A. A. Chaker, "Analysis of activity networks under uncertainty," Journal of Engineering Mechanics Division, vol. 101, Apr. 1975, pp. 373-387.

[2] K. Breitung, "Asymptotic Approximation for Multi-normal Integrals," Journal of Engineering Mechanics, vol. 110, Mar. 1984, pp. 357-366, doi: 10.1061/(ASCE)0733-9399(1984)110:3(357).

[3] C. G. Bucher, U. Bourgund, "A fast and efficient response surface approach for structural reliability problems," Structural Safety, vol. 7, Jan. 1990, pp. 57-66, doi: 10.1016/0167-4730(90)90012-E.

[4] S. Crandall, "First-crossing probabilities of the linear oscillator," Journal of Sound and Vibration, vol. 12, Jul. 1970, pp. 285-299, doi: 10.1016/0022-460X(70)90073-8.

[5] W. Fan, J. Wei, A. Ang, Z. Li, "Adaptive estimation of statistical moments of the responses of random systems," Probabilistic Engineering Mechanics, vol. 43, Jan. 2016, pp. 50-67, doi: 10.1016/j.probengmech.2015.10.005

[6] L. Faravelli, "A response surface approach for reliability analysis," Journal of Engineering Mechanics, vol. 115, Dec. 1989, pp. 27632781, doi: 10.1061/(ASCE)0733-9399(1989)115:12(2763).

[7] Y. Feng, F. Moses, "Optimum design, redundancy and reliability of structural systems," Computers \& Structures, vol. 24, Dec. 1986, pp 239-251, doi: 10.1016/0045-7949(86)90283-X.

[8] A. Hasofer and N. Lind, "Exact and Invariant Second Moment Code Format," Journal of Engineering Mechanics Division, vol. 100, Feb. 1974, pp. 111-121.

[9] J. Li, J. B. Chen, W. L. Fan, "The equivalent extreme-value event and evaluation of the structural system reliability," Structural Safety, vol. 29, Apr. 2007, pp. 112-131, doi: 10.1016/j.strusafe.2006.03.002.

[10] F. Moses, "Structural system reliability and optimization," Computers \& Structures, vol. 7, Apr. 1977, pp. 283-290, doi: 10.1016/00457949(77)90047-5.

[11] M. Rajashekhar and B. Ellingwood, "A new look at the response surface approach for reliability analysis," Structural Safety, vol. 12, Oct. 1993, pp. 205-220, doi: 10.1016/0167-4730(93)90003-J.

[12] S. Rice, "Mathematical Analysis of Random Noise," The Bell System Technical Journal, vol. 24, Jan. 1944, pp. 285-332, doi: 10.1002/j.1538-7305.1945.tb00453.x.

[13] P. Thoft-Christensen and J.D. Sørensen, "Reliability of structural systems with correlated elements," Applied Mathematical Modelling, vol. 6, Jun. 1982, pp. 171-178, doi: 10.1016/0307-904X(82)90006-3.

[14] P. Thoft-Christensen and Y. Murotsu, Application of Structural Systems Reliability Theory, 1986, Berlin, Heidelberg, New York, Tokyo: Springer-Verlag.

[15] Y. Zheng and P. K. Das, "Improved response surface method and its application to stiffened plate reliability analysis," Engineering Structure, Vol. 22, May. 2000, pp. 544-551, doi: 10.1016/S01410296(98)00136-9. 